not. Patient outcome was not known in a total of $11 \%$ of cases for reasons such as unavailability of notes, transfer of the patient to another hospital, or no follow up available from the GP. Overall, for both proactive and requested interventions, in $84 \%$ of the cases the advice given was acted on, in $8 \%$ it was not, and for $8 \%$ this information was not available. The relation between patient outcome and whether advice was acted on or not is shown in table 1 .

\section{Discussion}

This simple study provided much information on clinical activity by microbiologists in our hospital. It is noteworthy that in $84 \%$ of cases the advice given was accepted, whether re- quested or not, and was highly associated with patient improvement. The study highlighted areas where changes could be implemented such as education of clinical colleagues on appropriate use of antibiotics. The combination of a simple record form and a relational database system provides a means of performing further research and audit. It is hoped in the future to compare the patterns of clinical activity by microbiologists in other hospitals with different levels of departmental staffing, and to measure their impact on patient outcome.

I thank A McMillan for help with the database.

1 Mehtar S. How to cost and fund an infection control programme. $\mathcal{F}$ Hosp Infect 1993;25:57-69.

2 Daschner F. Cost-effectiveness in hospital infection controllessons for the 1990s. F Hosp Infect 1989;13:325-36.

\title{
Granulomatous disease in common variable immunodeficiency: effect on immunoglobulin replacement therapy and response to steroids and splenectomy
}

\author{
G P Spickett, J G Zhang, T Green, J Shrimankar
}

\section{Department of Immunology, Newcastle General Hospital, \\ Westgate Road, Newcastle upon Tyne NE4 6BE \\ G P Spickett \\ T Green}

Department of Histopathology J Shrimankar

\section{Department of} Immunology, The Medical School, University of Newcastle upon Tyne, Framlington Place, Newcastle upon Tyne NE2 4HH G P Spickett

J G Zhang

Correspondence to: Dr G P Spickett.

Accepted for publication 18 October 1995

\begin{abstract}
A 40 year old white woman with common variable immunodeficiency of four years duration presented with rapidly increasing splenomegaly. Despite high dose, weekly intravenous immunoglobulin, it was impossible to raise the trough serum IgG concentration to within the normal range. While waiting for a diagnostic splenectomy, low dose corticosteroids were started, leading to a decrease in the size of the spleen and an increase in the trough IgG concentration. Both spleen and liver showed non-caseating granulomas. Following splenectomy, the corticosteroids were tailed off and the trough IgG was maintained well into the normal range on a reduced, fortnightly dose of intravenous immunoglobulin and a low dose of oral corticosteroid.

(f Clin Pathol 1996;49:431-434)
\end{abstract}

Keywords: common variable immunodeficiency, intravenous immunoglobulin, granuloma.

Common variable immunodeficiency (CVID) is a primary immunodeficiency which may occur at any age and is manifest predominantly by antibody deficiency, leading to recurrent bacterial infections. ${ }^{1}$ The cause is unknown, but there are widespread immunological abnormalities. About $10 \%$ of patients lack B lymphocytes, and some of these patients have now been shown to carry the same molecular defect as has been described in patients with X-linked agammaglobulinaemia (XLA); $30 \%$ of patients with CVID may have splenomegaly. ${ }^{2}$ These patients tend to have a more severe disease clinically and more severe in vitro immunological abnormalities.

A granulomatous disease resembling sarcoidosis has been described in CVID. ${ }^{3-5}$ This condition is not found in XLA, indicating that it is not just a feature of severe or persistent infection, as the infection profiles for CVID and XLA are very similar. The disease behaves clinically very like sarcoidosis and may involve the lung, gut and nervous system. ${ }^{67}$ Both Kveim negative and Kveim positive variants have been described. ${ }^{47}$ The optimum treatment is unknown, but it is usually responsive to corticosteroids. It is rare for patients with CVID to undergo splenectomy as there is a reluctance to impose an additional immune deficiency on patients with a major pre-existing defect. However, splenectomy may be justified in cases where there is significant hypersplenism or concern about underlying lymphoma, the incidence of which is increased in patients with CVID. 


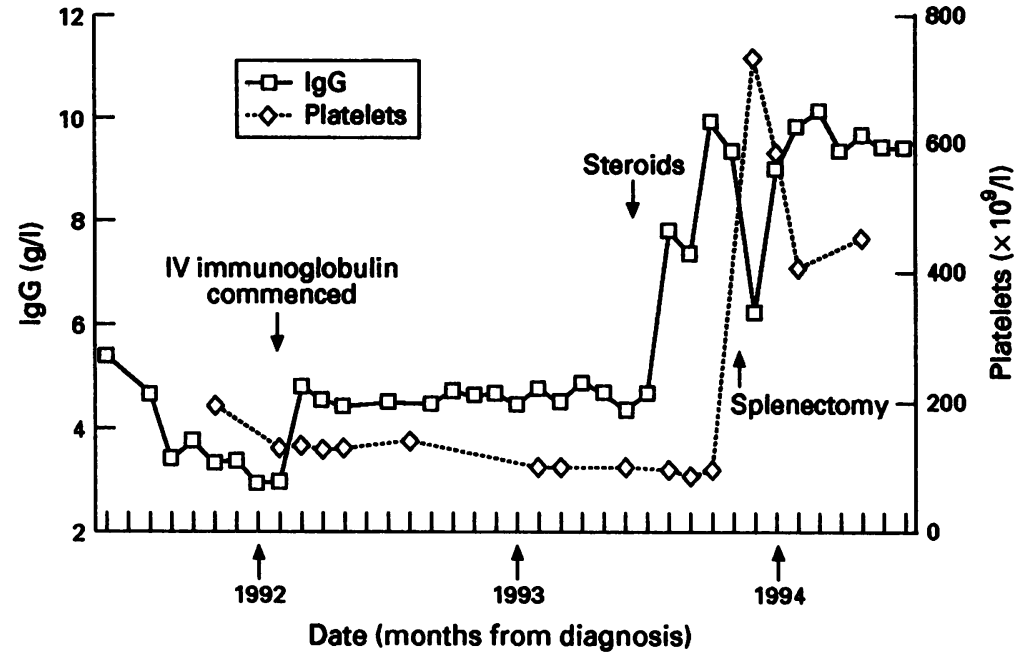

Figure 1 Plot of time (months from diagnosis) versus trough $\operatorname{IgG}(g / l)$ concentration and platelet count $\left(\times 10^{9} / 1\right)$.
In 1992, the patient's serum IgG continued to fall despite an increase in the intravenous immunoglobulin dose to $21 \mathrm{~g}$ every two weeks: the dose was changed to $9 \mathrm{~g} / 12 \mathrm{~g}$, alternating each week (fig 1). A prolonged bout of diarrhoea was investigated with stool cultures and barium meal and follow up: no cause was found and the diarrhoea settled spontaneously. Serum albumin was normal throughout, making significant gastrointestinal protein loss unlikely. However, on ultrasound the spleen had increased in size to $20 \mathrm{~cm}$. The platelet count, which had previously been normal, fell to $127 \times 10^{9} / 1$. There were bibasal crackles in both lung fields which were persistent; chest radiography showed patchy basal shadowing.

In 1993, the spleen continued to increase in size. The patient complained of noticeable malaise, shortness of breath on exertion and non-specific joint aches. Examination confirmed that the spleen had extended across the midline to the right iliac fossa, below the umbilicus. Coarse basal crackles were present in the lung fields. Her trough IgG was persistently low and her dose of intravenous immunoglobulin was increased to $12 \mathrm{~g} /$ week, with no effect on the trough IgG concentration. Pulmonary function tests showed a reduction of the gas transfer coefficient (Kco $61 \%$ predicted) and a chest radiograph showed more noticeable basal shadowing than previously. Repeat bone marrow examination showed no evidence of lymphoproliferative disease. Lymphocyte surface marker analysis showed a reduced $B$ cell number. A computed tomography scan confirmed splenomegaly and indicated some enlarged retrocaval and para-aortic lymph nodes. There was ground glass shadowing in the lower lobes of the lungs bilaterally, suggesting an interstitial infiltrate. A decision was made to remove the patient's spleen, as lymphoma could not be excluded. While awaiting splenectomy, the patient was treated with corticosteroids to improve her well-being and as an attempt to raise her platelet count. She was given $30 \mathrm{mg}$ prednisolone/day, reducing rapidly to $10 \mathrm{mg} /$ day. On this dose, her spleen shrank noticeably, her trough IgG rose to well into the normal range without any further change in her dose of intravenous immunoglobulin (fig 1), her Kco rose to $75 \%$ predicted and her respiratory symptoms disappeared. Her platelet count remained low at $83 \times 10^{9} / 1$.

At operation, the spleen was removed and open biopsy specimens were taken of liver and intra-abdominal lymph nodes. Following surgery, there was a typical post-splenectomy thrombocytosis which then settled to leave a platelet count at the top of the normal range. Her lymphopenia improved, with a lymphocyte count of $3.0 \times 10^{9} / 1$ and her trough IgG remained high (fig 1). She has been commenced on long term prophylactic penicillin and her steroid dose has been reduced to $5 \mathrm{mg} /$ day. She remains clinically well.

\section{Results}

Figure 1 shows the changes in the patient's serum trough IgG (taken before an infusion of to high dose oral iron. Investigation as part of a separate study (Davidson and Spickett, unpublished data) showed poor iron uptake and no evidence of Helicobacter pylori colonisation.

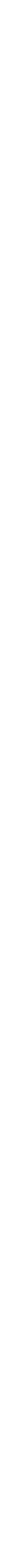




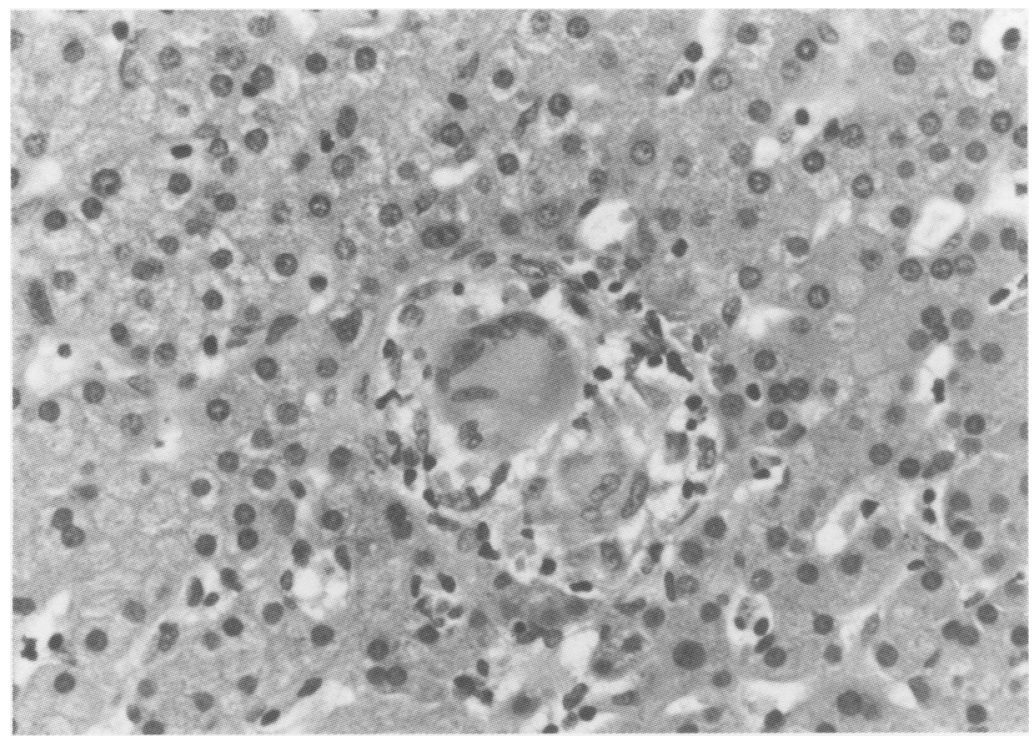

Figure 2 High power section of liver, showing a single, well defined granuloma with a multinucleate giant cell.
There is great reluctance to perform splenectomy in an already immunocompromised person and the recent Consensus document on the management of CVID suggests that splenectomy is undesirable. ${ }^{8}$ None the less, it may be necessary in order to exclude lymphoma or to deal with hypersplenism.

The histological features of the spleen in CVID are not well known. Weston et $a l^{9}$ have reported the appearances of spleens removed from three patients with CVID, all of whom were women. All three showed non-caseating epithelioid granulomas, which varied in both number and size; staining for non-specific esterase and lysozyme confirmed a macrophage origin, although one spleen contained granulomas with an admixture of epithelioid cells and fibroblasts. Total B cell numbers were reduced but lymphoid follicles and germinal centres could be demonstrated. Macrophage function is known to be abnormal in CVID and poor processing may contribute to granuloma formation; activated $\mathrm{T}$ cells are also required and $\mathrm{T}$ cell function is significantly impaired in CVID. ${ }^{10}$

Our patient had failed to achieve a satisshe was receiving weekly infusions. The rise in trough IgG concentration was immediate on commencing corticosteroids and was maintained even when the dose was reduced to $10 \mathrm{mg} /$ day before surgery. Following splenectomy, there was an immediate post-surgical drop, which recovered rapidly and subsequently the trough IgG concentration has been maintained at above $9 \mathrm{~g} / 1$ despite a reduction in her dosage to $18 \mathrm{~g}$ every two weeks (compared with a pre-surgery dose of $12 \mathrm{~g} /$ week), although she continues to take a very small dose of steroids.

The spleen was notably enlarged and weighed $1550 \mathrm{~g}$. On sectioning, it was congested and dispersed throughout the parenchyma were tiny nodules measuring between 2 and $4 \mathrm{~mm}$ in diameter. The histology of the spleen showed extensive non-caseating epithelioid and giant cell granulomas; there were lymphoid follicles of varying sizes in the white pulp with occasional germinal centres (not shown). The red pulp was congested with dilatated sinusoids. The liver showed normal architecture with a slight increase in inflammatory cell infiltrate. There were scattered focal collections of lymphocytes and a few well defined epithelioid and giant cell granulomas (fig 2). The lymph node showed sinus histiocytosis with depletion of lymphoid follicles (not shown). No organisms were seen.

\section{Discussion}

The association of granulomatous disease with CVID is well established and the early literature has been reviewed by Leen et al. ${ }^{4}$ However, the cause has never been established and although it is presumed to represent the response of an incompetent immune system to a persistent pathogen, no pathogen has ever been identified. It is suspected that the splenomegaly noted in many patients with CVID is related to granulomatous disease, although this is impossible to prove unless splenectomy is performed. factory trough IgG concentration despite high dose intravenous immunoglobulin, given weekly. Clearly, there was hypercatabolism of infused IgG, as there was no evidence to suggested increased loss through the urinary or gastrointestinal tracts. She did have a prolonged bout of diarrhoea early in her clinical course, but no cause was identified for this and there was no evidence to support a protein-losing enteropathy. Trough IgG remained low even after the diarrhoea had resolved. An alternative preparation was not tried. The effect of corticosteroids both on the splenomegaly and on the trough IgG concentration was surprising and the maintenance of the increase in the trough IgG concentration after splenectomy suggests that the spleen may be the site of the hypercatabolism of the IgG, although a small dose of steroids was continued after splenectomy. The corticosteroids probably had their effect through the notable reduction in the size of the spleen and also through alteration of macrophage function. Most immunologists have one or two patients in whom very large infused doses of intravenous immunoglobulin are required to achieve a satisfactory trough IgG concentration and prevent symptoms. Such patients may have macrophage mediated hypercatabolism, particularly if there is splenomegaly. The current case suggests that this may be associated with granulomatous disease. As quite small doses of corticosteroids seemed to be effective in suppressing the hypercatabolism, it may be worthwhile considering a trial of low dose corticosteroids in such patients.

We have elected to keep our patient on a small dose of corticosteroids, as she has definite evidence of granulomas in the liver and we strongly suspect that the pulmonary abnormalities are also due to granulomatous disease, as her respiratory symptoms resolved completely once corticosteroids were introduced. 
Dr J G Zhang is supported by the Sir Jules Thorn Charitable Trust.

1 Spickett GP, Misbah SA, Chapel HM. Primary antibody deficiency in adults. Lancet 1991;337:281-4.

2 Webster ADB, Farrant J, Hany $M$, North $M$, Toubi $E$, Beattie R. Clinical and cellular features of "Common Variable" Hypogammaglobulinaemia. EDS fournal of Immunology and Immunopharmacology 1991;11:32-5.

3 Crofts MW, Joyner MV, Sharp JC, Costello J, Vergani D. Sarcoidosis associated with combined immunodeficiency. Postgrad Med $¥$ 1980;56:263-5.

4 Leen CLS, Bath JCJL, Brettle RP, Yap PL. Sarcoidosis and primary hypogammaglobulinaemia: a report of two cases and a review of the literature. Sarcoidosis 1985;2:91-5.

5 Ortiz Arduán A, Sanz del Forcallo FJ, Carreira Delgado J, Garcia Delgado R, Ortiz Masllorens F, Castrillo Garcia
$\mathrm{JM}$, et al. Immunodeficiencia variable común y sarcoidosis. Rev Clin Esp 1992;190:75-80.

6 Misbah SA, Spickett GP, Esiri MM, Hughes JT, Matthews WB, Thompson RA, et al. Recurrent intra-cranial granulomata presenting as space-occupying lesions in a patient with common variable immunodeficiency. Postgrad Med $\mathcal{F}$ with common va

7 Hermaszewski RA, Webster ADB. Primary hypogammaglobulinaemia: a survey of clinical manifestations and complications. $Q \mathcal{F} \mathrm{Med}$ 1993;86:31-42.

8 Chapel HM. Consensus on diagnosis and management of primary antibody deficiencies. BMF 1994;308:581-5.

9 Weston J, Balfour BM, Tsohas W, English N, Farrant J, Webster ADB. Splenic lesions in hypogammaglobulinaemia. Adv Exp Biol Med 1993;329:437-41.

10 Spickett GP, Webster ADB, Farrant J. Cellular abnormalities in common variable immunodeficiency. Immunodefic Rev 1990;2:199-219.

\title{
Failure to kill Yersinia enterocolitica by plasma diluted to the concentration found in red cell units
}

\author{
A P Gibb, N Poling, W G Murphy
}

\begin{abstract}
The possibility that the use of additive solutions for red cell storage might impair the ability of plasma to kill Yersinia enterocolitica was investigated by studying killing of $Y$ enterocolitica by neat and diluted plasma. The ability of neat citrated plasma to kill complement sensitive organisms was lost at around $26 \%$, the dilution typically found in red cell units. These results should be considered in the light of evidence that killing in plasma is important in the protection of donated blood against growth of $Y$ enterocolitica, and the observation that the increase in frequency of transfusion reactions caused by $Y$ enterocolitica coincided with the widespread introduction of additive solutions. Taken together, these points support the suggestion that the introduction of additive solutions may have precipitated the problem of growth of $Y$ enterocolitica in stored blood.
\end{abstract}

(f Clin Pathol 1996;49:434-436)

Keywords: Yersinia enterocolitica, blood transfusion, red cell storage, complement.

Sepsis and death resulting from transfusion of blood containing large numbers of Yersinia enterocolitica was first reported in 1975, but not reported again until 1982. Since then, the number of cases reported has increased with a total of 10 cases reported by 1988 and at least 33 by $1992 .^{1-3}$ It is thought that the $Y$ enterocolitica found in donated blood originates as an asymptomatic bacteraemia in the donor. $Y$ enterocolitica is able to multiply at $4^{\circ} \mathrm{C}$, and can reach $10^{9} \mathrm{cfu} / \mathrm{ml}$ in blood at this temperature. Transfusion of blood containing large numbers of $Y$ enterocolitica has a high mortality $(21 / 33 ; 64 \%)$, but is rare with an estimated one death per nine million transfusions since 1986 in the USA.

The increase in the frequency of $Y$ enterocolitica induced sepsis following transfusion occurred at a time when the use of additive solutions for red cell storage was becoming more common. In the USA the use of additive solutions was approved by the Food and Drug Administration in 1983, and was adopted as standard in some states by $1985 .{ }^{4}$ This association may be significant as complement mediated killing in plasma is important in preventing the growth of $Y$ enterocolitica in donated blood, ${ }^{2}$ and as the use of additive solutions results in a reduced concentration of complement and other plasma components in the red cell storage medium. Details of red cell storage methods are not given in most case reports of $Y$ enterocolitica induced sepsis following transfusion. Red cells in additive solution were reported to be involved in some of the early cases, and in both of the cases reported from Edinburgh. ${ }^{5}$ The use of additive solutions can also be inferred from reference to a 42 day expiration date in the largest reported series. ${ }^{3}$

We have proposed ${ }^{2}$ that complement resistant phenotypes of $Y$ enterocolitica in the blood of the donor become complement sensitive during the first few hours after storage, because of the known effect of low temperature on the expression of complement resistance in $Y$ enterocolitica. In the presence of neat plasma the organisms are then killed, but in dilute plasma they could survive. Increased rate of cooling of blood, which may be a consequence of the use of red cell concentrates and additive solutions, also seems to contribute to survival 